\title{
Assessment of Retention of CAD-CAM Milled PEKK vs PEEK Double Crown-retained Removable Partial Dentures: A Randomized Clinical Trial
}

\author{
Omnia M Refai ${ }^{1}$, Noha H Nawar ${ }^{2}$, Ingy T Lebshtien $^{3}$
}

\begin{abstract}
Aim and objective: To assess and compare the dislodging force of double crown-retained removable partial dentures (RPDs) made from polyetherketoneketone (PEKK) and polyetheretherketone (PEEK) at insertion (baseline) and one year after clinical functional use.

Materials and methods: A total of 18 patients with maxillary symmetrical Kennedy class I with the second premolars being the last standing abutment were selected. The patients were randomly assigned into two groups according to the materials used for the construction of the double crown-retained RPD. Group I: Double crown-retained RPDs were made from PEKK. Group II: Double crown-retained RPDs were made from PEEK. For both groups, the primary copings were made from zirconia $\left(\mathrm{ZrO}_{2}\right)$. The dislodging force was measured using a digital force gauge at baseline and one year after clinical functional use. An independent $t$-test was used for intergroup comparisons and a paired $t$-test for intragroup comparisons. Results: when comparing both groups at baseline, there was no significant difference between the two groups, but after one year, Group I had a significantly higher dislodging force value than Group II. Individually, there was an increase in dislodging force in both groups one year after clinical functional use, which was statistically significant for Group I.

Conclusion: After clinical functional use, double crown-retained RPDs made from PEEK in combination with $\mathrm{ZrO}_{2}$ primary copings demonstrate a minimal increase in dislodging force, while those made of PEKK demonstrate a significant increase in dislodging force.

Clinical significance: All patients were satisfied with the retention and esthetics of their dentures. Polyetherketoneketone double crown-retained RPDs demonstrate better retention one year after clinical functional use.

Keywords: Computer-Aided Design-Computer-Aided Manufacturing, Denture retention, Polyetheretherketone, Randomized clinical trial, Telescopic attachments.

The Journal of Contemporary Dental Practice (2021): 10.5005/jp-journals-10024-3233
\end{abstract}

\section{INTRODUCTION}

Treatment options for partially edentulous patients with Kennedy Class I (bilateral free end saddle) can be removable partial denture (RPD), double crown-retained RPD, implant-retained or assisted prosthesis. Making the clinical decision for a suitable treatment option is critical for each case. ${ }^{1}$

Telescopic crown systems were innovated to increase the retention of RPD in the 20th century. A double crown-retained RPD consists of a primary coping, which is cemented to the abutment tooth, and a precisely fitted secondary crown, which is part of the framework of the subsequent RPD. ${ }^{2}$ Metal alloys are the most commonly accepted and used as telescopic crowns due to their excellent mechanical and physical properties. Gold alloys, cobalt chromium ( $\mathrm{CoCr}$ ), and titanium are examples of metal alloys used in telescopic crown systems. ${ }^{3}$

Also conventional lost wax technique was considered as a standard method for the fabrication of metal frameworks, it has many disadvantages like dimensional changes or errors in the casting procedure. ${ }^{4}$ The innovation of the computer-aided design-computer-aided manufacturing (CAD-CAM) fabrication technique has facilitated many construction steps, shortened the time of construction and eliminated many errors associated with dimensional changes and casting procedures. ${ }^{5}$ Additionally, this led to the use of new materials in the construction of telescopic crowns, like zirconia $\left(\mathrm{ZrO}_{2}\right)$, polyetheretherketone (PEEK), and polyetherketoneketone (PEKK). ${ }^{6}$

\footnotetext{
${ }^{1-3}$ Department of Oral and Maxillofacial Prosthodontics, Faculty of Dentistry, Ain Shams University, Cairo, Egypt
}

Corresponding Author: Omnia M Refai, Department of Oral and Maxillofacial Prosthodontics, Faculty of Dentistry, Ain Shams University, Cairo, Egypt, Phone: +201202420046, e-mail: dr.omnia.1990@gmail. com; omnia_refaie@dent.asu.edu.eg

How to cite this article: Refai OM, Nawar NH, Lebshtien IT. Assessment of Retention of CAD-CAM Milled PEKK vs PEEK Double Crownretained Removable Partial Dentures: A Randomized Clinical Trial. J Contemp Dent Pract 2021;22(11):1250-1256.

Source of support: Nil

Conflict of interest: None

Polyetheretherketone and PEKK are modifications of the main thermoplastic high-performance polymer group, polyaryletherketone (PAEK). ${ }^{7,8}$ Polyetheretherketone has high thermal and chemical stability as it has an aromatic backbone molecular chain, interconnected by ketone and ether functional groups. Additionally, PEEK has high biocompatibility, good mechanical properties, high temperature resistance, highly polished surface, low plaque affinity, acceptable low specific weight, and high bond strength with veneering composites and luting cements. ${ }^{7}$ Polyetherketoneketone is a liner thermoplastic polymer and consists of a benzene ring attached consecutively by ether or ketone groups. The second ketone group in PEKK ensures better

() The Author(s). 2021 Open Access This article is distributed under the terms of the Creative Commons Attribution 4.0 International License (https://creativecommons. org/licenses/by-nc/4.0/), which permits unrestricted use, distribution, and non-commercial reproduction in any medium, provided you give appropriate credit to the original author(s) and the source, provide a link to the Creative Commons license, and indicate if changes were made. The Creative Commons Public Domain Dedication waiver (http://creativecommons.org/publicdomain/zero/1.0/) applies to the data made available in this article, unless otherwise stated. 
mechanical and physical properties. ${ }^{9}$ Polyetherketoneketone has a higher compressive strength, excellent polishing ability, and a bone-like elastic modulus. ${ }^{8}$ Polyetherketoneketone has recently been applied in dentistry and is used in implant super-structures for fixed arch bridgework abutments and biomaterial, resin-based composite veneered substructures for bridges and removable restorations, for example, secondary structures via bars and telescopes. $8,10,11$

Zirconia is a ceramic material used for medical devices and its yttrium oxide stabilization demonstrates a combination of good esthetics, high mechanical strength, high biocompatibility, and resistance to wear. ${ }^{12}$ Zirconia has also been confirmed as a material for primary crowns in the double crown technique and has been used successfully as an alternative to gold alloy. ${ }^{13}$

A novel concept is to combine these two biocompatible materials, $\mathrm{ZrO}_{2}$ and PEEK, so as to obtain metal-free restorations such as telescopic attachments. The performance of PEEK as a double crown material has been thoroughly examined in vitro and in vivo, and it has been reported that it could be a suitable material for a telescopic crown. ${ }^{14-19} \mathrm{An}$ in vitro study reported that $\mathrm{ZrO}_{2}$ primary copings showed a comparable value at baseline when combined with either PEEK or electroformed secondary crowns. On the other hand, they showed a more constant retention force after 10 years of simulated aging. ${ }^{14}$ Another in vivo study has investigated PEEK as a double crown-retained RPD and found it produces an acceptable treatment option regarding bone loss. ${ }^{18}$ In a clinical study, they found that the combination of $\mathrm{ZrO}_{2}$ primary copings with PEEK complete overdenture retained on two implants is a biologically promising attachment for preserving peri-implant soft tissue health. ${ }^{19}$ Rare data are available about the combination of $\mathrm{ZrO}_{2}$ and PEKK telescopic attachments. An in vitro study has investigated the retention forces of secondary telescopic crowns made from PEKK in combination with $\mathrm{ZrO}_{2}$ primary crowns with a wear aging period equivalent to 10 years, and they found that retention increases with time. ${ }^{20}$

Despite the fact that many studies were conducted to evaluate the retention of PEEK secondary crowns in combination with $\mathrm{ZrO}_{2}$ primary copings, and it was shown the proper performance and suitability of these combinations, limited studies in the literature have analyzed the combination of PEKK secondary crowns in combination with $\mathrm{ZrO}_{2}$ primary copings. Therefore, this study aimed to assess the dislodging force of double crown-retained RPDs constructed from PEKK in combination with $\mathrm{ZrO}_{2}$ primary copings and to compare it with double crown-retained RPDs constructed from PEEK in combination with $\mathrm{ZrO}_{2}$ primary copings at the baseline and one year after clinical functional use. The null hypothesis of this study was that there would be no significant difference in dislodging force between PEKK and PEEK double crown-retained RPDs one year after clinical functional use.

\section{Materials and Methods}

\section{Study Design}

A randomized clinical trial was designed. Study timeline from enrolment to evaluation was demonstrated in CONSORT 2010 flow diagram (Flowchart 1).

\section{Sample Size}

A power analysis was designed to have adequate power to apply a two-sided statistical test of the null hypothesis that there is no difference would be found between tested groups regarding denture retention. By adopting an alpha and beta levels of 0.05 , that is,

Flowchart 1: Flow diagram according to CONSORT guidelines

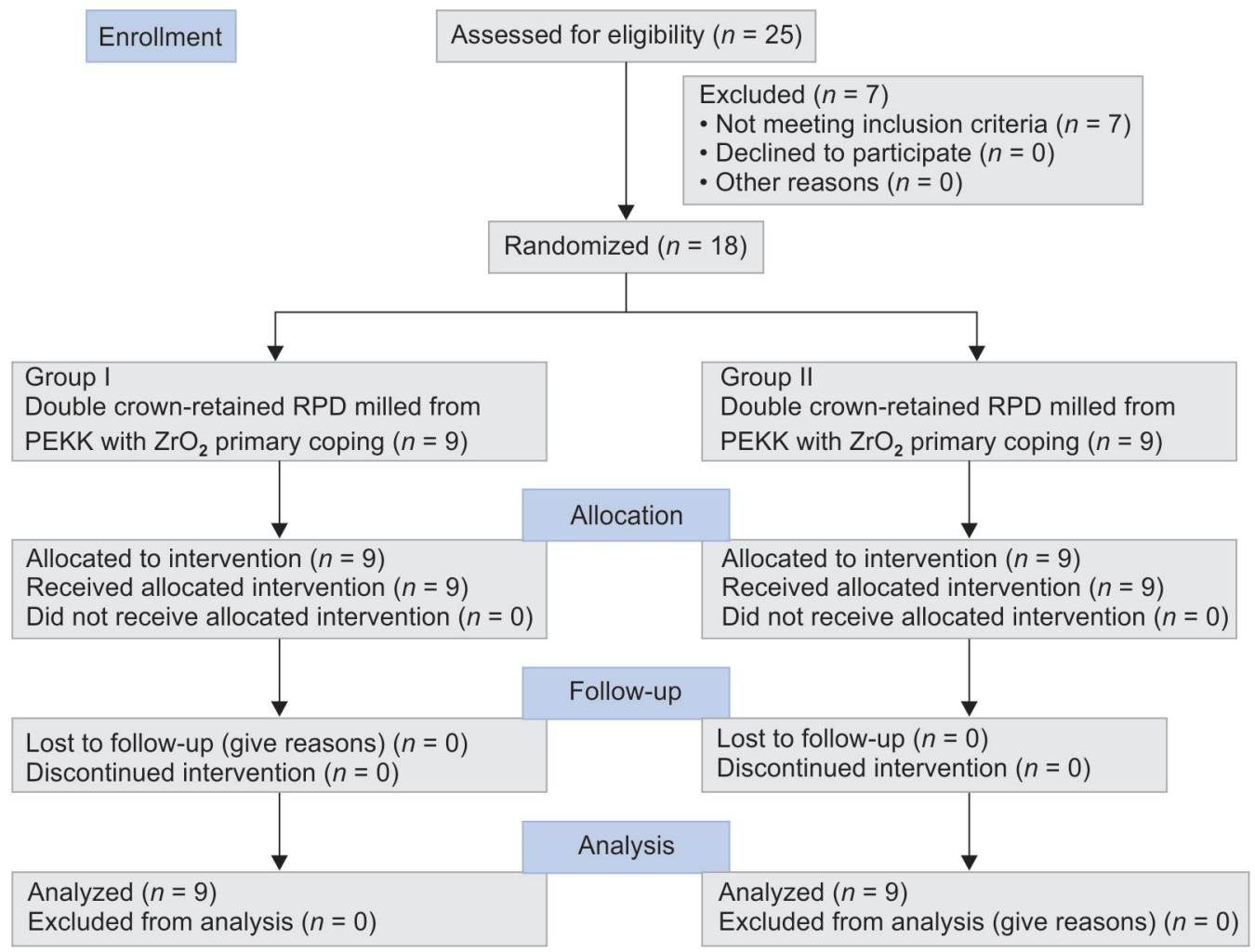


power $=95 \%$ and an effect size (d) of 2.29 calculated based on the results of a previous study. ${ }^{21}$ The predicted sample size $(n)$ was found to be a total of 14 cases. Sample size was increased to account for a dropout rate of $20 \%$ to be 18 cases (i.e., nine cases per group). Sample size calculation was performed using $\mathrm{G}^{*}$ Power version 3.1.9.7. ${ }^{22}$

\section{Patient Selection}

A total of 25 patients with maxillary Kennedy class I were diagnosed where 18 healthy male patients with an age range of $50-60$ years old who fulfilled the inclusion criteria were selected from the outpatient clinic of the Department of Prosthodontics, Faculty of Dentistry, Ain Shams University, to participate in this study. Detailed information about the study was given to all participants who signed a written consent for approval.

The protocol of the research was approved by the ethical committee of the Faculty of Dentistry, Ain Sham University (number of approval: FDASU-Rec ID041991). The period of this study started from April 2019 to May 2021. The follow-up period was one year after clinical functional use of double crown-retained RPD.

\section{Inclusion Criteria}

Patients were selected to have the following inclusion criteria: partially edentulous patients with maxillary symmetrical Kennedy class I (bilateral free end saddle) with the second premolars being the last standing abutments. Having an Angle's Class I maxillomandibular relationship with sufficient inter-arch space, good oral hygiene measures, and abutments in good periodontal condition.

\section{Exclusion Criteria}

Patients having the following criteria were excluded: abutments with Grade II or III mobility, an inadequate crown root ratio, and badly destructed abutments. Also, teeth with severe malalignment and patients with xerostomia. Additionally, patients exhibiting parafunctional habits or temporo-mandibular joint disorder, V-shaped palate and any sign of pathosis found on the edentulous ridge or hard palate.

\section{Patient Grouping (Randomization Process)}

The study participants were randomly assigned to one of two groups (I or II), each with nine participants, by a practitioner who was not involved in patient selection or treatment, was aware of the randomization sequence, and had access to the randomization lists stored on a password-protected laptop. The randomly generated codes using a research randomizer (Random.org) were placed in sequentially ordered, opaque, and sealed envelopes. Patients were asked to choose one of the envelopes; the practitioner, who was informed of the randomization process, was then asked to determine the group to which the patients were assigned. Participants in Group I received double crown-retained RPDs made from PEKK, while participants in Group II received double crownretained RPDs made from PEEK.

\section{Blinding}

In this study, participants were blinded by not revealing to them detail about the group they belonged to and the participants were not recalled on the same day. By concealing the group identities from the assessor, he was rendered blind.

\section{Patient Examination}

Clinical and radiographic examinations of the patients were performed to fulfil the pre-mentioned criteria.

\section{Patient Preparation}

Scaling and polishing of teeth was preformed, and the patient was instructed to perform restricted oral hygiene measures. Afterwards, a primary impression (CA37; Cavex Holland BV, Haarlem, Netherlands) was taken and the mounting of study casts on a semiadjustable articulator (A7 plus Articulator, Bio-Art, São Carlos, Brazil) was done via face-bow (Bio-Art Elite Face Bow, Bio-Art, São Carlos, Brazil) and provisional interocclusal records. Occlusal equilibration and adjustments were performed on the diagnostic casts and were used as a guide for intraoral modification.

\section{Abutments Preparation}

The last two abutments (first and second premolars) on each side were prepared with a deep chamfer finish line of $1.5 \mathrm{~mm}$, a 3- to 4-degree taper per wall, and an occlusal reduction of 2.5 $\mathrm{mm}$ was created. Retraction cord (Ultrapak ${ }^{\mathrm{TM}} \mathrm{E}$, Ultradent, South Jordan, USA) was applied for 5 minutes before taking a secondary impression (Elite HD+, Zhermack SpA, Polesine, Italy) with a putty wash technique. Casts were poured and occlusion blocks were fabricated on master casts. A face-bow record and an interocclusal record using wax wafer technique (CAVEX SET UP WAX, CAVEX, Haarlem, Netherlands) were performed for the mounting of the upper and lower casts.

\section{Designing and Construction of Primary Copings}

A gingivally trimmed master cast with removable dies was made from the duplication of the master cast. Then the gingivally trimmed cast, master cast, separate dies, and mounted casts were sprayed with a scanner spray (SCANTIST 3D Dental SCAN-SPRAY, scantist3d, Recklinghausen, Germany) and scanned with a desktop scanner (FREEDOM UHD, DOF, Seoul, Korea). The primary copings were designed with Exocad software (Exocad DentalCAD 2.4 Plovdiv, exocad $\mathrm{GmbH}$, Darmstadt, Germany) to have a common path of insertion and their cervical third was made parallel, while the remaining occlusal was made conus with a 3-degree taper. The thickness of the copings was kept at the minimum thickness, which was $1 \mathrm{~mm}$, with a 1-mm finish line, and the gap distance was set at $0.05 \mathrm{~mm}^{16}$ (Fig. 1).

The standard tessellation language (STL) file of the designed primary copings was converted to the CAM software (CORITEC ICAM V5 SMART, imes-icore ${ }^{\circledR} \mathrm{GmbH}$, Eiterfeld, Germany) for nesting and

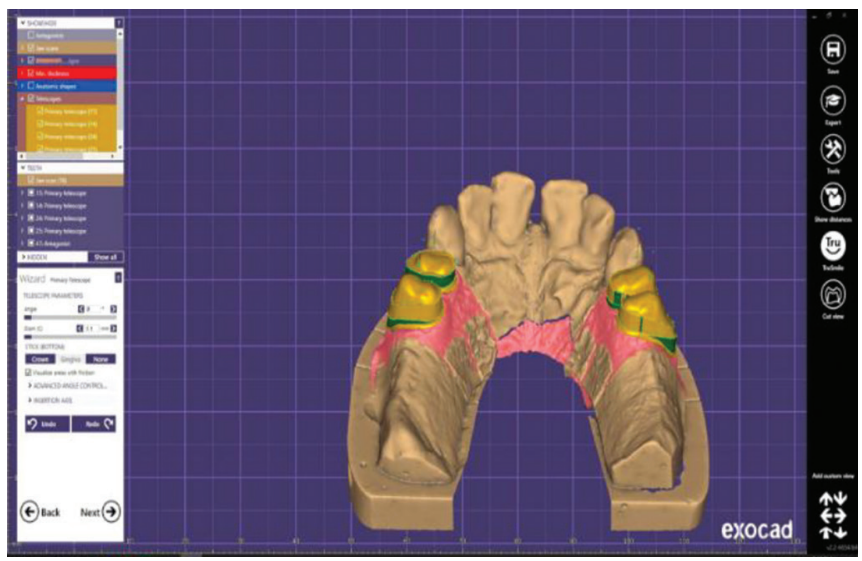

Fig. 1: Designing of the primary copings with their cervical third was made parallel, while the remaining occlusal was made conus with a 3-degree taper using Exocad software 
preparation to be milled from pre-sintered $\mathrm{ZrO}_{2}$ blank (Ceramill ${ }^{\circledR} \mathrm{zi}-$ AmannGirrbach AG, Koblach, Austria) by using a five-axis computer numeric control milling machine (Coritec 350i series, imes-icore ${ }^{\oplus}$ $\mathrm{GmbH}$, Eiterfeld, Germany). Afterwards, complete sintering in the furnace at $1,350-1,500^{\circ} \mathrm{C}$ to achieve its final shape. The fitting surface of each primary coping was sandblasted for 10 seconds with $110 \mu \mathrm{m}$ aluminum oxide particles. ${ }^{23}$

Try-in of primary copings was performed to ensure the marginal fit, accuracy, and $1.5 \mathrm{~mm}$ of occlusal clearance. After cementation of the primary copings with temporary cement (Cavex Temporary cement, Cavex, The Netherlands, Holand) a medium-consistency rubber base impression (THXOFLEX M, Zhermack SpA, Polesine, Italy) was taken, and the copings were returned to their position in the impression. A cast was poured and a trial denture base was fabricated. Another facebow record and interocclusal records were taken for the mounting of the upper and lower casts on the semiadjustable articulator.

\section{Designing of the Secondary Crowns and RPD Framework}

The mounted casts and the upper cast with primary copings were sprayed and scanned with the desktop scanner. The STL files were transferred to obtain a virtual model, which was digitally surveyed to determine the most acceptable path of insertion and to design secondary copings with a minimal axial and occlusal thickness of 1 $\mathrm{mm}$ and an occlusal space of 0.3 to $0.5 \mathrm{~mm}$ between the inner and outer crowns. ${ }^{24,25} \mathrm{~A}$ palatal plate major connector, denture base meshwork, and an external finish line were drawn ${ }^{26}$ (Fig. 2). The STL file of the designed secondary copings and RPD framework was transferred to the CAM software for preparation to be milled from PEKK (Pekkton ${ }^{\oplus i v o r y, ~ C e n d r e s ~+~ M e ́ t a u x ~ S A, ~ B i e l / B i e n n e, ~}$ Switzerland) or PEEK (ceramill ${ }^{\circledR}$ peek-AmannGirrbach AG, Koblach, Austria) blank according to the group (Fig. 3).

Try-in of the milled framework was preformed and shade selection for the veneering of secondary copings with high impact composite polymer filled with a microceramic layer (Novo.lign Veeners, Bredent, Senden, Germany) was performed. Then another bite registration was taken in centric relation for mounting of the maxillary and mandibular casts. Then setting-up of the remaining posterior teeth and waxing-up were completed.

\section{Processing and Insertion of the Double Crown-retained RPD}

Try-in of the waxed-up denture was preformed to check occlusion and fit of the denture. Then processing of the denture using heat-cured acrylic resin (Vertex Rapid Simplified, Vertex Dental, Soesterberg, Netherlands) via a conventional long polymerization cycle was performed, and it was finished and polished in the usual manner.

Primary copings were permanently cemented to their abutments via a self-etching and self-adhesive dual-cure resin cement (TOTALCEM, ITENA, Villepinte, France) according to the manufacturer's instructions. Afterwards, the double crown-retained RPD was delivered and any occlusal adjustments were performed (Fig. 4). Patients were instructed to perform oral and denture hygiene measures.

\section{Evaluation of Dislodging Force}

A metallic bar was attached to the occlusal surface of the denture at the geometric center of the denture using self-curing acrylic resin (Acrostone Cold Cure, Acrostone Dental \& Medical Supplies, Cairo, Egypt). A notch was made in the inner surface of the bar at the midline, then the double crown-retained RPD was immersed

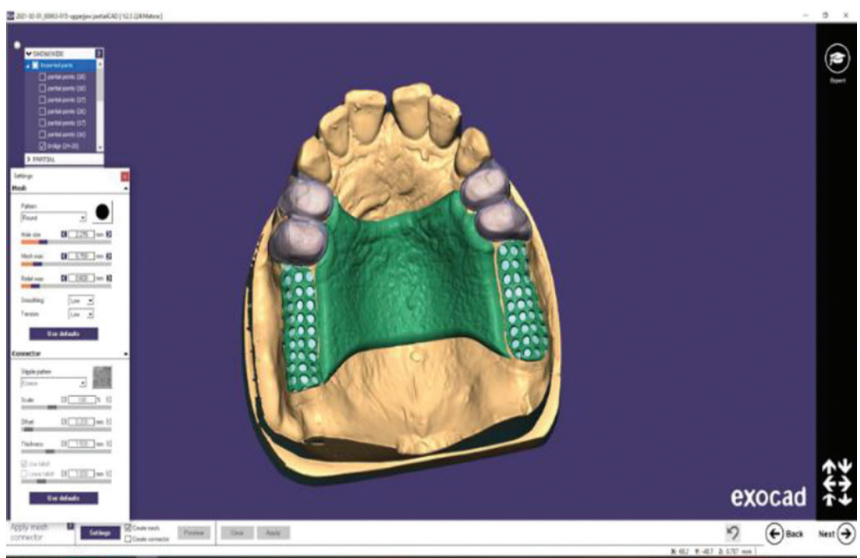

Fig. 2: Design of the double crown-retained RPD framework with palatal plate major connector using Exocad software

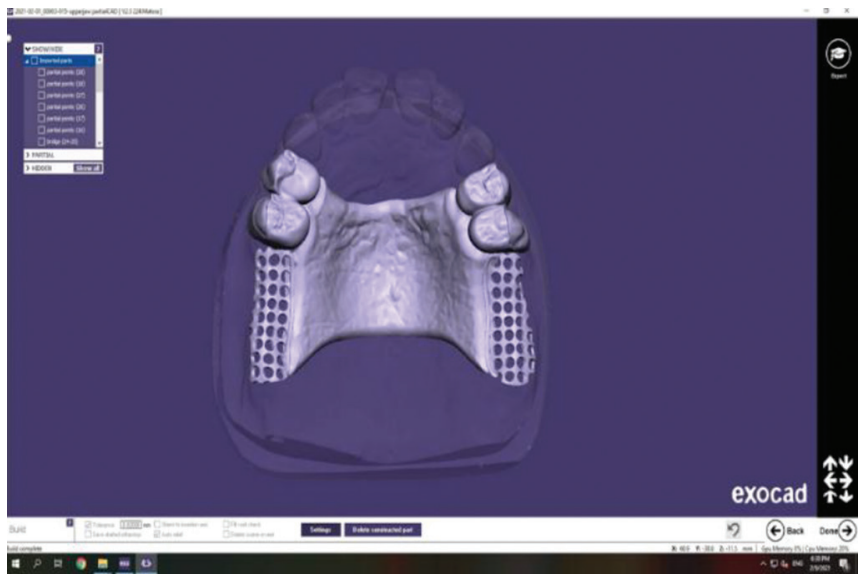

Fig. 3: STL file of the final design of the PEKK framework before milling

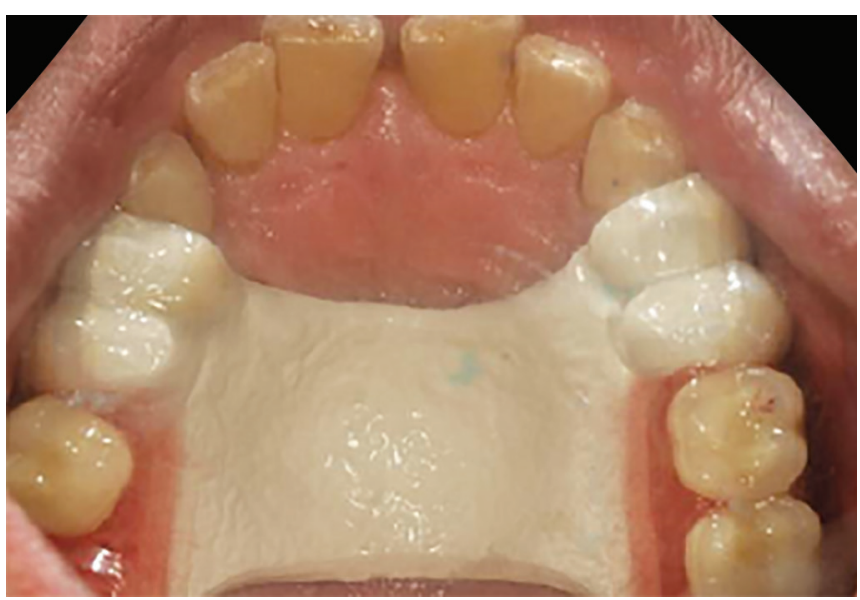

Fig. 4: Delivery of PEKK double crown-retained RPD after occlusal adjustments

in water for 10 minutes before measuring the dislodging force. Dislodging force was measured by using a digital force gauge (HF-100 Digital Force Gauge, Jinan Hensgrand Instrumentation Co., Ltd., Jinan, China). The digital force gauge was prepared first. The 
unit of measurement was selected to be in Newton and the peak hold option was selected. The desired adapter tension hook was attached, and the display before each measurement was adjusted to zero via the zero buttons. The patient was seated in an upright position, and then he was instructed to tilt his head backward till the applied dislodging force was nearly perpendicular to the denture. The dislodging force was applied till the denture was pulled out, and it was measured five times at 5-minute intervals, and an average value was recorded. 27,28

The evaluation of dislodging force was performed for each double crown-retained RPD by an independent assessor at insertion (baseline) and one year after clinical functional use. At the recall appointment (one year after clinical functional use), patients were asked about their experience with retention and esthetic of their dentures.

\section{Statistical Analysis}

Numerical data were tested for normality using Shapiro-Wilk's test. Data were parametric so they were presented as mean and standard deviation values and were analyzed using independent $t$-test for intergroup comparisons and paired $t$-test for intragroup comparisons. The significance level was set at $p<0.05$ within all tests. Statistical analysis was performed with $R$ statistical analysis software version 4.1.2 for Windows (R Core Team (2021)).

\section{Results \\ Demographic Data}

The sample size calculations revealed that 14 people were needed for this study, but the number was increased to 18 to account for the loss of follow-up. As a result, a total of 18 people were recruited at the beginning of the study. The cases were assigned equally and randomly into two groups, I and II (i.e., nine cases each). During the follow-up phase, no individuals dropped out of the study. In Group $\mathrm{I}$, the average age of the participants was $54.00 \pm 2.74$ years, while in Group II, it was $56.00 \pm 2.74$ years. There were no significant differences between both groups regarding the age $(p=0.1409)$ of the participants.

\section{Intergroup Comparison}

Results of inter- and intragroup comparisons of denture dislodging forces presented in Table 1 showed that when comparing both groups (intergroup comparison), at baseline there was no significant difference between both groups ( $p=0.077)$, while after one year, Group I had a significantly higher dislodging force value than Group II ( $p=0.009)$ (Table 1).

\section{Intragroup Comparison}

The intragroup comparison showed that in Group I there was a significant increase in dislodging force value after one year $(p=0.006)$, while in Group II there was no significant difference between values measured at different intervals $(p=0.228)$. In addition, there was no

Table 1: Inter- and intragroup comparisons of dislodging force $(N)$

\begin{tabular}{lccc}
\hline & \multicolumn{2}{c}{ Dislodging force $(\mathrm{N})($ mean $\pm S D)$} & \\
\cline { 2 - 3 } Interval & Group I & Group II & p value \\
\hline Baseline & $19.52 \pm 0.88^{\mathrm{Ab}}$ & $18.67 \pm 0.53^{\mathrm{Aa}}$ & 0.077 \\
After one year & $25.02 \pm 2.76^{\mathrm{Aa}}$ & $20.10 \pm 2.45^{\mathrm{Ba}}$ & $0.009^{*}$ \\
Difference & $4.00 \pm 1.46^{\mathrm{A}}$ & $2.58 \pm 0.81^{\mathrm{A}}$ & 0.074 \\
\hline
\end{tabular}

Means with different upper and lowercase letters within the same horizontal row and vertical column respectively are statically different. *Significant $p<0.05$ significant difference in the change in dislodging force after one year between both groups $(p=0.074)$. All patients were satisfied with the retention and esthetics of their dentures (Table 1).

\section{Discussion}

The recent introduction of digital techniques into dentistry has facilitated the construction of double crown-retained RPDs. Additionally, it has expanded the range of materials that can be utilized for double crown-retained RPD production, including high thermoplastic PEKK and PEEK materials. Telescopic attachments are used to increase RPD retention. However, maintaining their retentive values over a long period of time is critical for the treatment's success. ${ }^{14-17}$ This study was conducted to assess and compare the dislodging force of double crownretained RPD constructed from PEKK and PEEK in combination with $\mathrm{ZrO}_{2}$ primary copings at the baseline and one year after clinical functional use.

The null hypothesis of this study was rejected as there was a significant difference in dislodging force between PEKK and PEEK double crown-retained RPD one year after clinical functional use.

This study used PEEK because of its many advantages, including its high biocompatibility, good mechanical properties, high temperature resistance, chemical stability, highly polished surface, low plaque affinity, low specific weight, and the ability to fabricate lighter metal-free RPDs that are esthetically acceptable, as well as its high bond strength with veneering composites and luting cements. ${ }^{7}$ Polyetherketoneketone was used in this study due to its carbon fiber reinforced with extra ketone group, which shows excellent properties, including high thermal stability, low moisture absorption, excellent flammability resistance, high mechanical properties, low specific weight, shock-absorbing ability, high toughness, and tensile modulus. It is worth noting that only a few studies have been performed on PEKK previously. ${ }^{8,11,20}$ In order to improve the efficiency of the telescopic system, a combination of primary copings made of $\mathrm{ZrO}_{2}$ and secondary crowns made of PEEK or PEKK was used in this study. This combination has been shown to have high biocompatibility and retention, as well as an improved ability to absorb occlusal forcers. ${ }^{20,29}$

The telescopic crown design used in this study was a resilient double crown, which allows minor lateral movement between the crowns and minimizes transmission of stresses to the abutments due to the presence of clearance fit between the outer and inner crown. This design was proved to be suitable for tooth-mucosa supported dentures. ${ }^{24,25}$

In this study, secondary crowns were veneered with a high impact polymer composite filled with a micro ceramic layer because it was determined that a reliable bond strength existed between PAEK polymers and composite veneering resins. ${ }^{30}$

Most studies evaluating dislodging forces were performed in a laboratory setting to allow for the application of pure vertical dislodging forces perpendicular to the occlusal plane and to avoid non-axial dislodging of the dentures if it was measured clinically due to the presence of the opposing jaw. As a result, non-axial dislodging is common, which does not represent retention forces but rather stability forces. A digital force gauge was used in this study to measure the clinical dislodging force values because it combines the advantages of in vitro and in vivo retention measurement. In the presence of an oral environment such as humidity, saliva, and temperature, this device applies pure vertical force perpendicular to the occlusal plane. ${ }^{27,28,31}$ 
At the baseline, the dislodging force values for both groups were nearly the same. This is due to the fact that both are from the same PAEK-based polymer family and were constructed by milling technique. ${ }^{7-9,32}$

It has been revealed that using hard and wear-resistant materials for primary copings and less hard materials for secondary crowns may be advantageous. Minimal changes will happen to the primary coping, which is designed in accordance with the dentist's treatment plan, and the adaptation between both crowns will be accomplished through permanent changes to the secondary crown, which explains the increase in dislodging force in both groups during functional clinical use. ${ }^{33} \mathrm{~A}$ significant increase in dislodging force appeared in Group I, and this is may be explained by the flexibility and ductility of PEKK, resulting in a good marginal fit and mechanical adaptation between the primary copings and secondary crowns, so a strong wedging action occurred between the two crowns after functional use. ${ }^{20}$

The results of the dislodging force of Group II showed a nonsignificant increase between baseline and one year after clinical functional use. This was supported by a study conducted in vitro by Schubert et al. ${ }^{14}$ Polyetheretherketone secondary crowns against $\mathrm{ZrO}_{2}$ primary copings exhibited a stable retention force over time of simulated aging, and this was explained by the excellent physical, chemical, and mechanical properties of PEEK. ${ }^{14}$

The results of dislodging force value were significantly increased one year after clinical functional use in Group I. These results were agreed with a study conducted by Kotthaus et al. ${ }^{20}$ Polyetherketoneketone secondary telescopic crowns against $\mathrm{ZrO}_{2}$ primary copings exhibited an increase in dislodging force after simulated aging.

Despite the fact that CAD-CAM technology is useful for the construction of double crown-retained RPDs with a lower incidence of human errors and the production of a proper retentive and esthetic prosthesis, the milling technique requires expensive new equipment and produces a lot of material waste. ${ }^{34}$

The limitations of this study are that the study results reflect only one year of clinical functional use, so further long-term studies are needed to investigate the retention of PEKK and PEEK doublecrown-retained RPD. In addition, different investigations were not performed, like the assessment of stress induced on the supporting structure by both groups and the assessment of abutment bone level after clinical functional use.

Future research can be directed comparing the two groups over a long-term follow-up and to assess the stress induced on the supporting structure, soft tissue level around the abutments and bone level of the abutments after clinical functional use.

\section{Conclusion}

Based on the findings of the study, it is possible to conclude that the fabrication of double crown-retained RPDs from PEEK in combination with $\mathrm{ZrO}_{2}$ primary copings results in a minimal increase in dislodging force, whereas those from PEKK results in a significant increase in dislodging force one year after clinical functional use.

\section{Clinical Significance}

All patients were satisfied with the retention and esthetics of their PEKK and PEEK double crown-retained RPDs. One year after clinical functional use, the PEKK double crown-retained RPDs demonstrate better retention.

\section{Declaration of Interest}

The authors declare no conflicts of interest, financial or personal relationships with any organization that may influence the results of this study.

\section{References}

1. Öwall B, Käyser AF, Carlsson GE. Prosthodontics: principles and management strategies. 7th ed. London: Mosby-Wolfe1996.9p.

2. Hakkoum MA, Wazir G. Telescopic Denture. Open Dent J 2018;12(1):246-254. DOI: 10.2174/1874210601812010246.

3. Wataha JC. Alloys for Prosthodontic Restorations. J Prosthet Dent 2002;87(4):351-363. DOI: 10.1067/mpr.2002.123817.

4. Diwan R, Talic Y, Omar N, et al. Pattern Waxes and Inaccuracies in Fixed and Removable Partial Denture Castings. J Prosthet Dent 1997;77(5):553-555. DOI: 10.1016/s0022-3913(97)70152-5.

5. Janeva NM, Kovacevska G, Elencevski S, et al. Advantages of CAD/ CAM versus Conventional Complete Dentures-A Review. Open Access Maced J Med Sci 2018;6(8):1498-1502. DOI: 10.3889/oamjms.2018.308.

6. Kamel A, Badr A, Fekry G, et al. Parameters Affecting the Retention Force of CAD/CAM Telescopic Crowns: A Focused Review of In Vitro Studies. J Clin Med 2021;10(19):4429. DOI: 10.3390/jcm10194429.

7. Papathanasiou I, Kamposiora P, Papavasiliou G, et al. The Use of PEEK in Digital Prosthodontics: A Narrative Review. BMC Oral Health 2020;20(1):217. DOI: 10.1186/s12903-020-01202-7.

8. Alqurashi $\mathrm{H}$, Khurshid Z, Syed AUY, et al. Polyetherketoneketone (PEKK): An Emerging Biomaterial for Oral Implants and Dental Prostheses. J Adv Res 2020;28:87-95. DOI: 10.1016/ j.jare.2020.09.004.

9. Brown T, Bao B, Kilpela T, et al. An In Vitro Biotribological Assessment of NUBAC, a Polyetheretherketone-On-Polyetheretherketone Articulating Nucleus Replacement Device: Methodology and Results from a Series of Wear Tests Using Different Motion Profiles, Test Frequencies, and Environmental Conditions. Spine J 2010;35(26): 774-781. DOI: 10.1097/BRS.0b013e3181d59e45.

10. Park C, Jun J, Park W, et al. Use of Polyaryletherketone (PAEK) Based Polymer for Implant-Supported Telescopic Overdenture: A Case Report. J Adv Prosthodont 2017;9(1):74-76. DOI: 10.4047/jap.2017.9.1.74.

11. Shetty S, Shenoy K, Shetty R, et al. PEKK (Polyetherketoneketone) as a Prosthetic Material- A review. Int J Recent Sci 2018;9(4):25724-25726. Available from: http://dx.doi.org/10.24327/ijrsr.2018.0904.1917.

12. Gautam C, Joyner J, Gautam A, et al. Zirconia Based Dental Ceramics: Structure, Mechanical Properties, Biocompatibility and Applications. Dalton Trans 2016;45(48):19194-19215. DOI: 10.1039/c6dt03484e.

13. Beuer F, Edelhoff D, Gernet W, et al. Parameters Affecting Retentive Force of Electroformed Double-Crown Systems. Clin Oral Investig 2010;14(2):129-135. DOI: 10.1007/s00784-009-0271-7.

14. Schubert O, Reitmaier J, Schweiger J, et al. Retentive Force of PEEK Secondary Crowns on Zirconia Primary Crowns Over Time. Clin Oral Investig 2019;23:2331-2338 DOI: 10.1007/s00784-018-2657-x.

15. Emera RM, Abelkhalek S, Rashed M. Periodic Retention Evaluation of Two Implants Retained Complete Mandibular Overdenture with Zirconia-PEEK Telescopic Attachments. J Dent Sci 2019;18:15-24. DOI: 10.9790/0853-1803141524.

16. Emera RM, Altonbary G, Elbashir S. Comparison between All Zirconia, All PEEK, and Zirconia-PEEK Telescopic Attachments for Two Implants Retained Mandibular Complete Overdentures: In Vitro Stress Analysis Study. J Dent Imp 2019;9:24.15. DOI: 10.4103/jdi.jdi_6_19.

17. Emera RM, Elgamal M, Albadwei M. Surface wear of All Zicronia, All PEEK and Zirconia-Peek Telescopic Attachments for Two Implants Retained Mandibular Complete Overdentures. In-Vitro study using scanning electron microscope. J Dent Sci 2019;18:59-68.DOI: 10.9790/0853-1802095665.

18. Elsarrif HR, Mohamed SH, Sabet ME. Effect of Different Techniques of CAD/CAM designed BIOHPP Frameworks on the Supporting Structures of Kennedy Class I Telescopic Partial Denture Cases. Egypt Dent J 2021 Oct;67(4):3393-3402. DOI: 10.21608/edj.2021.76015.1677.0. 
19. Emera RM, Khalifa A, Ahmed WS, et al. Clinical Outcome of Two Implants Retained Complete Mandibular Overdenture with ZirconiaPEEK Telescopic Attachment. Int J Curr Adv Res 2019;8:19540-19546. DOI: 10.24327/ijcar.2019.

20. Kotthaus M, Hasan I, Keilig L, et al. Investigation of the Retention Forces of Secondary Telescopic Crowns Made from Pekkton ${ }^{\circledast}$ Ivory in Combination with Primary Crowns Made from Four Different Dental Alloys: An In Vitro Study. Biomed Tech 2019;64(5):555-62. DOI: 10.1515/bmt-2018-0167.

21. El Mekawy N, Gad E. Retentive Force and Surface Roughness of Partial Removable Frameworks Fabricated from Conventional Chrome-Cobalt and CAD/CAM Modified Polyetheretherketone Materials (Within-Subject Evaluation). Egypt Dent J 2016;62: 5055-5062. DOI: 10.30476/edj.2016.76015.1677.0.

22. Faul, Franz, et al. G* Power 3: A Flexible Statistical Power Analysis Program for the Social, Behavioral, and Biomedical Sciences. Behav Res Methods 2007;39.2:175-191. DOI: 10.3758/BRM.41.4.1149.

23. Su N, Yue L, Liao Y, et al. The Effect of Various Sandblasting Conditions on Surface Changes of Dental Zirconia and Shear Bond Strength Between Zirconia Core and Indirect Composite Resin. J Adv Prosthodont 2015;7(3):214-223. DOI: 10.4047/jap.2015.7.3.214.

24. Langer A. Telescope Retainers and Their Clinical Application.J Prosthet Dent 1980;44(5):516-522. DOI: 10.1016/0022-3913(80)90070-0.

25. Wenz HJ, Hertrampf K, Lehmann KM. Clinical Longevity of Removable Partial Dentures Retained by Telescopic Crowns: Outcome of the Double Crown with Clearance Fit. Int J Prosthodont 2000;19(3): 207-213. PMID: 11484566.

26. Tamimi F, Almufleh B, Caron E, et al. Digital Removable Partial Dentures. Clin Dent Rev 2020;4(9):24-29. DOI: 10.1007/s41894-02000074-y.
27. Maryod W, Taha E. Retention of Removable Partial Denture Fabricated by Digital Designing and 3D Printing Technology-A Cross over Study. Advan Dent \& Oral Health 2019;10(3):555-789. DOI: 10.19080/ ADOH.2019.10.555789.

28. Faty MA, Sabet ME, Thabet YG. A Comparison of Denture Base Retention and Adaptation between CAD-CAM and Conventional Fabrication Techniques. Int J Prosthodont 2021 DOI: 10.11607/ijp.7193.

29. Merk S, Wagner C, Stock V, et al. Suitability of Secondary PEEK Telescopic Crowns on Zirconia Primary Crowns: The Influence of Fabrication Method and Taper. Materials (Basel) 2016;9(11):908-912. DOI: $10.3390 / \mathrm{ma} 9110908$.

30. Stawarczyk B, Taufall S, Roos M, et al. Bonding of Composite Resins to PEEK: The Influence of Adhesive Systems and Air-abrasion Parameters. Clin Oral Investig 2018;22(2):763-771. DOI: 10.1007/ s00784-017-2151-x.

31. Colón A, Kotwal K, Mangelsdorff AD. Analysis of the Posterior Palatal Seal and the Palatal form as Related to the Retention of Complete Dentures. J Prosthet Dent 1982;47(1):23-27. DOI: 10.1016/00223913(82)90237-2.

32. Wang $Y$, Wang $P, X u$ Q, et al. Synthesis and Properties of Novel Copolymers of Poly (Ether Ketone Biphenyl Ketone Ether Ketone Ketone) and Poly (Ether Ketone Sulfone Amide). J Polym Res 2014;21(8):533. DOI: 10.1002/pi.6181.

33. Bayer S, Zuziak W, Kraus D, et al. Conical Crowns with Electroplated Gold Copings: Retention Force Changes Caused by Wear and Combined Off-Axial Load. Clin Oral Implants Res 2011;22(3):323-329. DOI: 10.1111/j.1600-0501.2010.02003.x.

34. Beuer F, Schweiger J, Edelhoff D. Digital Dentistry: An Overview of Recent Developments for CAD/CAM Generated Restorations. Br Dent J 2008;204(9):505-511. DOI: 10.1038/sj.bdj.2008.350. 